\title{
Corticotrophin-releasing hormone type 1 receptor gene $(C R H R 1)$ variants predict posttraumatic stress disorder onset and course in pediatric injury patients
}

\author{
Ananda B. Amstadter ${ }^{\mathrm{a}, *}$, Nicole R. Nugent ${ }^{\mathrm{b}}$, Bao-Zhu Yang ${ }^{\mathrm{c}}$, Alisa Miller ${ }^{\mathrm{d}}$, Richie Siburian ${ }^{\mathrm{e}}$, \\ Priya Moorjanie ${ }^{e}$, Stephen Haddad ${ }^{\mathrm{e}}$, Aditi Basu ${ }^{\mathrm{e}}$, Jesen Fagerness ${ }^{\mathrm{e}}$, Glenn Saxe ${ }^{\mathrm{d}, \mathrm{f}}$, \\ Jordan W. Smollere,f and Karestan C. Koenen ${ }^{\mathrm{g}}$ \\ ${ }^{a}$ Department of Psychiatry, Virginia Institute for Psychiatric and Behavioral Genetics, Virginia Commonwealth \\ University, Richmond, VA, USA \\ ${ }^{\mathrm{b}}$ Department of Psychiatry, Brown Medical School and Rhode Island Hospital, Providence, RI, USA \\ ${ }^{\mathrm{c}}$ Department of Psychiatry, Division of Human Genetics, Yale University School of Medicine, New Haven, \\ Connecticut, CT, USA \\ ${ }^{\mathrm{d}}$ Department of Psychiatry, Boston Children's Hospital, Boston, MA, USA \\ ${ }^{\mathrm{e}}$ Psychiatric Genetics Program in Mood and Anxiety Disorders and Psychiatric and Neurodevelopmental Genetics \\ Unit, Center for Human Genetic Research, Massachusetts General Hospital, Boston, MA, USA \\ ${ }^{\mathrm{f}}$ Harvard Medical School, Boston, MA, USA \\ ${ }^{\mathrm{g}}$ Departments of Society, Human Development and Health and Epidemiology, Harvard School of Public Health, \\ Boston, MA, USA and the Harvard Center on the Developing Child, Cambridge, MA, USA
}

\begin{abstract}
Posttraumatic stress disorder (PTSD) is a common and disabling anxiety disorder that may occur in the aftermath of exposure to potentially traumatic life events. PTSD is moderately heritable, but few specific molecular variants accounting for this heritability have been identified. Genes regulating the hypothalamic-pituitary-adrenal (HPA) axis, such as corticotrophinreleasing hormone type 1 receptor gene (CRHRl), have been implicated in traumatic-stress related phenotypes but have yet to be studied in relation to PTSD. The present study sought to examine the relation between 9 single nucleotide polymorphisms (SNPs) in the CRHR1 gene and posttraumatic stress symptoms in a prospective study of pediatric injury patients $(n=103)$ who were first assessed in the acute aftermath of their injury at the hospital. Results indicated that multiple SNPs were associated with acute symptoms at a univariate level, and after correction for multiple testing, rs 12944712 was significantly related to acute PTSD symptoms. Longitudinal latent growth curve analyses suggest that rs12944712 is also related to both acute symptom level and trajectory of symptoms over time. The present study adds support for the role of CRHR1 in the stress response following potentially traumatic event exposure in youth. It should be noted that the sample size in this study was small, and therefore statistical power was low; following, results from this study should be considered preliminary. Although results are not definitive, the findings from this study warrant future replication studies on how variation in this gene relates to response to traumatic event exposure in youth.
\end{abstract}

Keywords: Posttraumatic stress disorder, CRHR1, hypothalamic-pituitary-adrenal axis, genetic, injury

\footnotetext{
${ }^{*}$ Corresponding author: Ananda B. Amstadter, PhD; Virginia Institute for Psychiatric and Behavioral Genetics, Virginia Commonwealth University, Department of Psychiatry, 800 E. Leigh Street, PO Box 980126, Richmond, VA 23298-0126, USA. Tel.: +1 804828 8129; Fax: +1 804828 8801; E-mail: abamstadter@vcu.edu.
} 


\section{Introduction}

Stressful experiences involve the recruitment of the body's major stress systems, including the hypothalamic-pituitary-adrenal (HPA) axis. The HPA axis, activated by corticotrophin-releasing hormone (CRH), regulates the release of stress hormones such as cortisol [1]. In many individuals, this normative stress response is followed by a return to baseline once the stressor has passed. However, a subset of individuals evidence alterations in this initial stress response and followed by the chronic dysregulation of the HPA axis characteristic of traumatic stress related phenotypes, including posttraumatic stress disorder (PTSD) [2]. There are a number of key findings suggesting HPA axis dysregulation that are exhibited in those with PTSD (e.g., elevated cerebral spinal fluid corticotrophin releasing hormone, enhanced suppression of cortisol, lower glucocorticoid receptors in lymphocytes), all of which are consistent with the notion of a sensitized HPA axis in individuals with PTSD [for review see 2]. Therefore, there is reason to hypothesize that genes regulating the HPA axis would be relevant to study in relation to posttraumatic stress symptom trajectory. Notably, however, these findings suggest that the HPA axis may be dysregulated in individuals with PTSD, but they are not specific to PTSD. Many phenotypes are common following exposure to traumatic events, such as depression and other anxiety disorders, and many of these disorders also evidence HPA axis dysregulation.

Although there are numerous candidate genes with potential influence on the HPA axis [3], CRH system genes play a critical role in modulation of HPA stress reactivity [4-7]. As such, these genes may influence acute and/or chronic alterations in HPA axis functioning. Although a total of thirty investigations have examined candidate genes and PTSD [8], only two studies have examined whether polymorphisms believed to influence HPA axis functioning are related to PTSD [9,10], and no prior studies of PTSD have examined $C R H$ genes. The present investigation examines the association between nine genetic markers spanning the CRH receptor 1 (CRHRl) gene (rs11657992, rs12936181, rs12944712, rs17690314, rs17763658, rs242942, rs4074461, rs4458044, rs11657992) and longitudinal trajectories of PTSD symptoms in pediatric injury patients.

To date, only one study of candidate genes and PTSD has focused on children [11]. Animal and human studies support the developmental sensitivity of stress effects on the HPA axis [12,13]. Indeed, age and develop- mental stage at the time of the trauma and at the time of later assessment have been linked to different patterns of both acute and chronic HPA axis alterations $[14,15]$. For example, whereas low levels of peritrauma cortisol predict later development of PTSD in adults [16], high peritrauma cortisol levels predict subsequent development of PTSD in children [17-19]. Interestingly, longitudinal assessments comparing trauma-exposed youth who did versus did not develop PTSD found that, although acute measures of acute salivary cortisol were predictive of subsequent development of PTSD (measured at 1 and 6 months), salivary cortisol levels and rhythm normalized by 6 months [19]. This pattern of changing direction in cortisol-PTSD associations was also found in an investigation of youth assessed within a year of their trauma compared with youth assessed more than one year post-trauma [20]; whereas PTSD symptoms were linearly and positively associated with cortisol in recently traumatized youth, symptoms were negatively associated with cortisol in youth whose experiences occurred more than a year prior. These investigations highlight key considerations for researchers interested in understanding the relationship between the HPA axis (as indicated by cortisol) and PTSD: (1) the association between cortisol and subsequent PTSD differs in youth and adults and (2) the direction of the association between cortisol and PTSD in youth changes as time since trauma elapses. Thus, while the genetic predictors of associations between HPA axis functioning and PTSD are unknown, it is clear that both age at time of trauma and duration of time since trauma are important considerations in traumatic stress research.

Recently, variation in the CRHR1 gene (CRHR1 rs110402 and rs242924) was found to interact with a history of childhood maltreatment to predict adult cortisol response to the dexamethasone/CRH test [7]; in both markers, the minor (i.e., less common) alleles were found to protect against the effects of childhood maltreatment, with maltreated participants homozygous (GG) for the major allele found to evidence increased stress reactivity relative to maltreated participants who were carriers of the minor alleles. This finding was partially replicated in a second investigation of one of the variants (rs110402), in which the minor allele was associated with decreased cortisol response to the dexamethasone/CRH test in males, but not females, reporting childhood trauma [21]. Notably, these effects were observed in participants with adverse early life experiences.

Numerous studies of depression provide support for the CRHRl gene as a moderator of psychopathology 
following exposure to early life stressors. For example, Bradley and colleagues (2008) reported an interaction between genetic markers spanning $C R H R I$ and childhood abuse in prediction of adult depression [22]. Seven out of ten markers spanning the gene showed significant interactions with childhood abuse in the prediction of depression, with rs110402 and rs7209436 significant even after correction for multiple tests. Consistent with prior research, participants experiencing childhood abuse and possessing the GG rs110402 genotype were at greatest risk for depression. Examination of common haplotypes of $C R H R I$ revealed a protective effect of a TAT haplotype formed by three CRHRI variants (rs7209436, rs110402, rs242924). Providing even further support for these findings, the results were replicated with an independent sample that was ethnically distinct from the original sample. Polanczyk and colleagues [23] attempted to replicate and extend the Bradley et al. investigation using data from two longitudinal cohort studies. Although findings from one cohort replicated the protective nature of the TAT haplotype (rs7209436, rs110402, and rs242924) in the prediction of depression, results of the second cohort did not support the expected effects. However both of these investigations assessed both early life stress and the psychiatric outcome many years after these experiences, introducing the potential for recall bias and limiting researchers' ability to prospectively examine psychobiological developmental processes in response to trauma. Additionally, although both depression and PTSD are stress-sensitive conditions characterized by alterations of the HPA axis, the patterns of HPA activity and reactivity found in PTSD diverge markedly from the patterns found in depression [24].

Although there is considerable theoretical support for the importance of CRHRI in the development of psychopathology following exposure to significant early life stress, no extant studies have examined CRHRI markers in the prediction of PTSD following trauma exposure during childhood. Indeed, no investigations to date have examined CRHRI as related to PTSD in any sample. Another limitation of extant research is the absence of studies that examine the influence of genes on both onset of symptoms and symptom course. As we have argued elsewhere [25], genes implicated in the development or onset of symptoms of PTSD may differ from genes implicated in the maintenance or course of symptoms of PTSD. Unlike previous cross-sectional investigations of PTSD candidate genes, the present investigation involves recruitment and assessment of participants within hours of trauma exposure followed by subsequent assessments of symptoms of PTSD at 3 months, 12 months, and 18 months. Thus, the present investigation can model not only the effects of genotype at a single time point but also influence of genotype over time. Consistent with cross-sectional research, we expected that the minor allele would be protective at the intercept (within hours of injury). However, as no prior studies have assessed the influence of candidate genes on PTSD symptom course, we did not make a priori hypotheses about the effects of CRHRI on symptom course.

\section{Methods and materials}

\subsection{Participants}

Participants were 103 children who were sequentially admitted to an inner-city hospital for injuries between April 2002 and January 2004 [26,27]. All children aged 7-18 admitted to the hospital with an injury were eligible to participate unless they or their parents did not speak sufficient English to complete the study instruments, had a Glasgow Coma Scale equal to or less than 7 at the time of their admission, or lived more than 2 hours away from the hospital (complicating follow up interviews). Participant characteristics are displayed in Table 1.

\subsection{Enrollment and acute assessment procedure}

The families of children who were admitted to the hospital with an injury were introduced to the study by a master's level research associate, once the child was deemed medically stable by the attending surgeon (e.g., they did not have a delirium, an active infection and were not receiving mechanical ventilation). Families were told that the study was investigating a child's coping following injury. All families were informed that participation in the study was voluntary and to decline participation would not affect their quality of care. Written informed consent was obtained from the parents and child after the researcher gave a comprehensive description of the study. The study was approved by the Institutional Review Board and treatment of human subjects adhered to established guidelines. The research associate interviewed the child and the primary caregiver during the initial hospital stay using the psychometric assessment package described below. Every effort was made to interview the child and caregiver separately, however the sensitive and complicated na- 
Table 1

Participant characteristics

\begin{tabular}{lc}
\hline & N (\%)/M (S.D.) \\
\hline Sex & \\
$\quad$ Male & $76(73.8)$ \\
Female & $27(26.2)$ \\
Age & \\
$7-8$ & $8(7.8)$ \\
$9-10$ & $7(6.8)$ \\
$11-12$ & $9(8.7)$ \\
$13-14$ & $11(10.7)$ \\
$15-16$ & $31(30.1)$ \\
$17-18$ & $37(35.9)$ \\
Self-Reported Race & \\
Non-Hispanic White & $42(40.8)$ \\
Non-Hispanic Black & $47(45.6)$ \\
Other & $14(13.6)$ \\
Injury type & \\
$\quad$ Violent & $29(28.2)$ \\
$\quad$ Non-Violent & $74(71.8)$ \\
Injury Severity Score & $8.91(6.91)$ \\
Number of Days in the Hospital & $5.90(5.78)$ \\
Acute UCLA Posttraumatic Stress Disorder Reaction Index & $24.09(12.21)$ \\
3-month UCLA Posttraumatic Stress Disorder Reaction Index & $21.68(12.59)$ \\
12-month UCLA Posttraumatic Stress Disorder Reaction Index & $20.20(11.60)$ \\
18-month UCLA Posttraumatic Stress Disorder Reaction Index & $16.85(10.26)$ \\
\hline & \\
\hline &
\end{tabular}

ture of the hospital stay and setting (e.g., grief, anxiety, doctor consultations, nurses visits, vitals being taken, etc.) precluded this in some cases. Each participant provided a saliva sample using a standard mouthwash protocol for DNA extraction [28]. Participants were paid $\$ 50$ for participation in the acute assessment, and they were also paid $\$ 25$ (and transportation costs) to complete follow-up assessments.

\subsection{DNA isolation}

Buccal DNA samples were obtained from each subject via mouthwash. DNA was isolated via standard procedures using the Gentra DNA isolation kit (Gentra Systems, Minneapolis MN).

\subsection{SNP selection and genotyping}

SNP selection was determined using the Phase II HAPMAP [29]. We used the aggressive tagging option (2- or 3-haplotype tagging of SNPs) of Tagger implemented in the program Haploview [30,31]. We further enriched our SNP selection by including SNPs implicated in anxiety phenotypes from the published literature. Genotyping of SNP markers was performed by mass spectrometry through use of the iPlex assay (Sequenom). The major steps in this process included the following: primer design using SpectroDESIGNER software; DNA amplification by PCR; post- amplification removal of the phosphate groups from the unincorporated dNTPs using shrimp alkaline phosphotase; primer extension reactions for allele differentiation, salt removal using ion-exchange resin; and SpectroCHIP plating/analysis by mass spectrometry.

\subsection{Outcome measure}

Posttraumatic stress symptoms were assessed during the acute hospitalization, and at 3-, 12-, and 18month follow-ups using the University of California at Los Angeles Child Post-traumatic Stress Disorder Reaction Index (UCLA PTSD-RI; [32-34]). The UCLA PTSD-RI is a 20-item semi-structured interview that assesses posttraumatic symptoms in children. Children are asked to rate the frequency of their posttraumatic symptoms on a 5-point Likert scale from $0=$ "never" to $4=$ " most of the time". The measure is most often used continuously, but a clinical cutoff of 38 can also be used [34]. The UCLA PTSD RI is internally consistent, with Cronbach's alpha in a disaster sample was reported to be 0.92 , and the measure has demonstrated excellent test-retest reliability $(0.84)$ [35]. The measure has convergent validity coefficients ranging from 0.70 (in comparison with the PTSD Module of the Schedule for Affective Disorders and Schizophrenia for School-Age Children) to 0.83 (in comparison to the Child and Adolescent Version of the Clinicianadministered PTSD Scale). Using a cutoff score of 38 , 
sensitivity has been found to be 0.93 and specificity to be 0.87 in detecting accurate PTSD diagnoses [33]. The UCLA PTSD-RI is one of the most widely used measures of PTSD symptoms in children [34].

\subsection{Covariates}

Race/ethnicity information was gathered via selfreport. For analytic purposes, participants were characterized as Non-Hispanic white, non-Hispanic black, or other. Sex was defined as male or female, and age at admission was measured in years. Injury Severity Score (ISS) [36] is a well-validated index of the injury severity. The ISS is related to the likelihood of survival after injury and is determined by rating the severity of injury for six body areas (i.e., head, neck, face, chest, abdomen, extremity and external) on a five-point scale known as the abbreviated injury scale (AIS). The AIS ranges from 1 (minor injury) to 5 (critical injury). The numerical score represents the degree of life threat associated with the anatomical injury. The ISS is derived from the sum of the squares of the AIS score with a range of 0-75. For patients with multiple traumas the three most severe injures are squared and summed. A trained trauma nurse coordinator assigned the ISS score of the participants in this study. Number of days in the hospital and whether the participant had experienced a violent (e.g., was shot, stabbed or physically assaulted) versus non-violent injury (e.g., car accident) were extracted from medical records.

\subsection{Statistical analyses}

Given the low number of children with probable PTSD at baseline $(n=18,17.5 \%)$ and at 3-month follow-up ( $n=7,6.8 \%$ ) the UCLA PTSD-RI was used continuously to maximize power. First, single SNP analyses were conducted in PLINK [37] to determine if any of the nine SNPs genotyped within CRHRI were associated at a bivariate level to UCLA PTSDRI total score. Also using PLINK, we derived empirical p-values using permutation tests $(10,000$ permutations $[38,39])$. We adjusted for multiple testing using a Bonferroni correction.

Finally, the longitudinal influence of CRHRI rs1294 4712 genotype was modeled using latent growth modeling (LGM) in MPlus. Analyses explored linear growth as well a quadratic slope factor [40]. Parameter estimates were conducted using maximum likelihood estimation with robust standard errors (MLR), which permits application of the missingness option, allowing retention of all participants on endogenous variables. Minimum missingness in the present study was 0.33 , suggesting sufficient covariance coverage for a reliable model [41]. Trajectories of linear time were parameterized using months, with the intercept indicated at the acute in-hospital assessment to permit examination of the influence of rs12944712 on initial symptom onset. On the basis of prior research supporting additive protective effects of the minor allele of rs12944712, the effects of rs12944712 were modeled in assumption of an additive genetic effect, with the alleles coded for symptom risk conferred (i.e., major alleles (G) were coded to indicate greater "dose," AA $=0, A G=1$, GG $=2$ ). To control for potential population stratification self-reported racial/ethnic status was included as a covariate. All analyses also covaried for key individuallevel influences (i.e., age, sex) as well as injury-related factors (i.e., injury severity, days in hospital, whether injury was sustained due to violence).

\section{Results}

\subsection{Descriptive analyses}

Sample characteristics are presented in Table 1. Participants' racial/ethnic status was reported as: nonHispanic white $(n=42,40.8 \%)$, non-Hispanic black ( $n=47,45.6 \%)$, or other $(n=14,13.6 \%)$. In regard to sex, $73.8 \%$ ( $n=76$ ) of the participants were male, and the remaining $26.2 \%(n=27)$ were female. The average age of participants was 14.63 years (S.D. = 3.18). With regard to injury characteristics, the average number of days spent in the hospital was 5.90 (S.D. = 5.78). The majority of the injuries were non-violent in nature $(71.8 \%, n=74)$, with the remaining $28.3 \%$ $(n=29)$ being violent in nature. The average ISS score was 8.91 (S.D. = 6.91). With regard to UCLA PTSD-RI scores, the average score was 24.09 (S.D. = 12.21) at the hospital, 21.68 (S.D. = 12.59) at 3-month follow-up, 20.20(S.D. = 11.60) at 12 month follow-up, and 16.85 (S.D. $=10.26$ ) at the 18 -month follow-up.

Racial/ethnic status was not related to UCLA PTSDRI scores at any of the assessment time points, suggesting that even if we did not control for racial/ethnic status, population stratification was not a possible cause of bias in results. Female sex was related to PTSD symptoms at the acute assessment, 3-month follow-up, and female sex was marginally related to PTSD symptoms at the other follow-up assessments. Age of participant was not related to UCLA PTSD-RI total score 
Table 2

List of tested CRHR1 SNPS. Their positions on human chromosome 17, hardy-weinberg equilibrium test $\mathrm{p}$ value, minor/major alleles, minor allele frequency and call rate

\begin{tabular}{lccccc}
\hline dbSNP marker & Chromosomal position & Hardy-Weinberg p-value & Minor/major alleles & Minor allele frequency & Call rate\% \\
\hline rs4074461 & 41210922 & 1.00 & $\mathrm{G} / \mathrm{T}$ & 0.48 & 94.3 \\
rs12944712 & 41226918 & 1.00 & $\mathrm{~A} / \mathrm{G}$ & 0.33 & 94.3 \\
rs4458044 & 41229498 & 0.37 & $\mathrm{C} / \mathrm{G}$ & 0.13 & 93.2 \\
rs12936181 & 41232250 & 0.37 & $\mathrm{C} / \mathrm{T}$ & 0.22 & 91.7 \\
rs242942 & 41247413 & 1.00 & $\mathrm{~T} / \mathrm{G}$ & 0.16 & 94.3 \\
rs17763104 & 41261576 & 0.61 & $\mathrm{~A} / \mathrm{G}$ & 0.12 & 94.3 \\
rs17690314 & 41275664 & 0.35 & $\mathrm{G} / \mathrm{T}$ & 0.12 & 94.3 \\
rs17763658 & 41278420 & 0.39 & $\mathrm{~A} / \mathrm{G}$ & 0.07 & 94.3 \\
\hline
\end{tabular}

at any time point. In regard to injury characteristics, violent assaults were related to PTSD-RI score at 12and 18-month follow-up assessments. No other injury characteristics were correlated with UCLA PTSD-RI score as any time point.

\subsection{Genetic analyses}

One marker, rs11657992 was excluded from analyses due to its low minor allele frequency (0.0115). The remaining SNP identifications, their locations, the Hardy-Weinberg Equilibrium test $P$ - values, minor/major alleles, minor allele frequencies, and call rate percentages are shown in Table 2. Call rates for SNPs ranged from 91 to $95 \%$ which included samples that failed all assays and for which DNA quality was inferior. All SNPs were in Hardy-Weinberg Equilibrium.

The gene encoding CRHRI is located on chromosome 17q21.31 and contains 13 exons spanning $51 \mathrm{~kb}$. To capture genetic variation across the CRHRl locus, we selected a set of 9 SNP located in a 71-kb region with an average intermarker distance of $9.6-\mathrm{kb}$ (note that one SNP was removed from analyses). We used Haploview [30] to determine the LD structure of the SNPs within the CRHRl gene which is presented in Fig. 1. These 8 SNPs capture 46 of the 79 (58\%) alleles at an $\mathrm{R}^{2}>0.8$ using a pair-wise tagging approach based on the HAPMAP [29] CEPH sample set, and the SNPs capture $62 \%$ of the $C R H R 1$ alleles at an $\mathrm{r}^{\wedge} 2$ $\geqslant 0.80$ using an aggressive 2 - and 3-haplotype. We identified two different pairs of blocks using the Four Gamete Rule and the Solid Spine of LD definitions in Haploview. Of the 103 children from whom we received DNA, 4 were removed for low genotyping $(<$ $90 \%)$.

\subsection{Association analyses}

We first conducted single SNP analyses to determine which markers were associated with UCLA PTSD-RI score at the acute hospital assessment. As displayed in Table 3, three SNPs were significantly related to PTSD symptom frequency (rs4074461, rs12944712, rs17763104) using empirical p-values. With Bonferroni correction, one of the eight SNPs (rs12944712) remained significantly associated with PTSD symptom frequency (see Table 3) and was therefore examined in the latent growth analysis below.

\subsection{Latent growth analyses}

The influence of rs12944712 in CRHRl on the trajectory of PTSD symptoms was examined using latent growth curve analysis, controlling for empirically- and theoretically-determined covariates including individual-level influences (i.e., age, sex, race) as well as injury-related factors (i.e., injury severity, days in hospital, whether injury was sustained due to violence); all participants with complete data on exogenous variables were retained in this analysis $(N=87)$. Using random coefficients regression (Raudenbush and Bryk, 2002) and maximum likelihood estimation procedures, a trend for each participant's symptoms of PTSD over each time point (in hospital, 3 months, 12 months, and 18 months) was modeled. Participant intercept (acute symptoms of PTSD) and both linear and quadratic slope (symptoms of PTSD over time) were predicted by covariates and rs12944712. Estimation difficulties were encountered in attempts to add nonlinear terms. Findings revealed significant effects of rs12944712 on both the intercept of acute PTSD symptoms as well as the linear slope of PTSD symptoms over time. Specifically, having an increasing number of $\mathrm{G}$ alleles was significantly associated with more acute symptoms of PTSD, Estimate $=5.77, \mathrm{SE}$ (Estimate) $=$ 1.43 , z-statistic $=3.35$, p-value $<0.001$. Additionally, having an increasing number of $\mathrm{G}$ alleles was associated with a significantly greater decline in PTSD symptoms over time, Estimate $=-0.42$, SE (Estimate $)=$ 
Table 3

Single SNP analyses in relation to acute UCLA PTSD-RI score

\begin{tabular}{lrllrl}
\hline dbSNP marker & \multicolumn{1}{c}{$\beta$} & S.E. & \multicolumn{1}{c}{$\mathrm{R}^{2}$} & \multicolumn{1}{c}{$t$} & Empirical p-value \\
\hline rs4074461 & 4.10 & 1.71 & 0.06 & 2.40 & 0.02 \\
rs12944712 & -5.36 & 1.83 & 0.08 & -2.94 & $0.005^{*}$ \\
rs4458044 & -1.53 & 2.48 & 0.004 & -0.62 & 1.00 \\
rs12936181 & 2.07 & 2.10 & 0.01 & 0.99 & 0.42 \\
rs242942 & -1.00 & 2.44 & 0.002 & -0.40 & 0.78 \\
rs17763104 & -6.66 & 2.71 & 0.07 & -2.46 & 0.02 \\
rs17690314 & -5.41 & 2.89 & 0.04 & -1.87 & 0.08 \\
rs17763658 & 3.90 & 3.27 & 0.02 & 1.19 & 0.26 \\
\hline
\end{tabular}

${ }^{*}$ significant after Bonferroni correction $(p=0.05 / 8=0.00625)$.

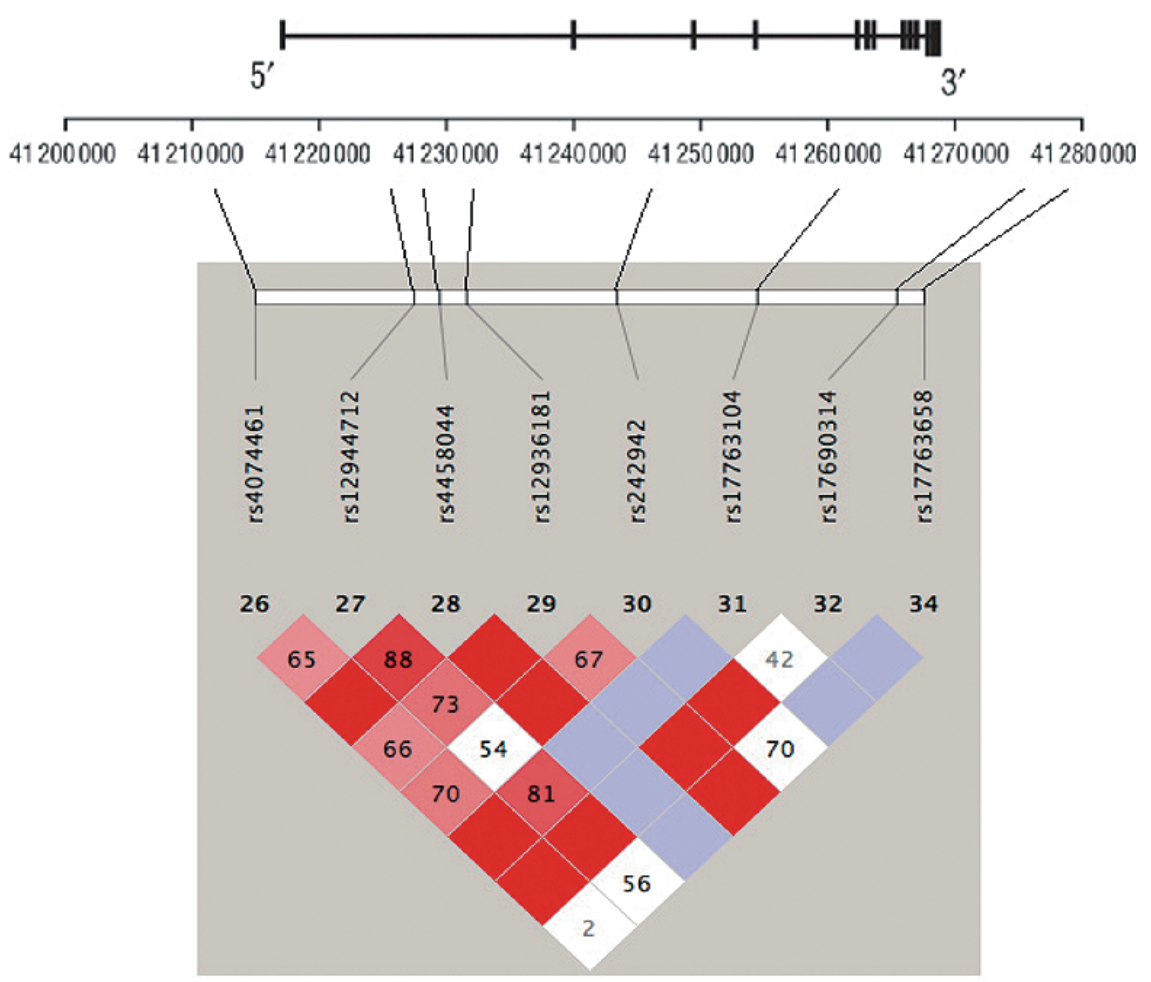

Fig. 1. The position of the CRHRl gene and its exons (filled rectangles) on chromosome 17 as well as a linkage disequilibrium (LD) plot of all tested SNPs using $\mathrm{r} 2$ as the measure of $\mathrm{LD}$.

0.11 , z-statistic $=-3.74$, p-value $<0.001$. As shown in Table 4, female gender was associated with greater levels of acute PTSD symptoms and having sustained a violent injury was associated with greater symptoms of PTSD over time. Unadjusted mean values for these data are shown in Fig. 2.

\section{Discussion}

Data reported in this study yielded two main findings. First, results suggest that polymorphisms in the CRHRI gene, which is involved in activation of the
HPA pathway, are related to acute PTSD symptoms in pediatric injury patients. Second, latent growth curve analyses modeling the trajectory of PTSD symptoms over time suggest that CRHRl variation is associated with both onset and course of PTSD symptoms. This study is unique in numerous ways; it is not only the first study to examine CRHRI variation in relation to PTSD symptoms, but it is also the first longitudinal examination of genetic variation on PTSD symptom trajectory. Furthermore, of the 30 candidate gene studies of PTSD [8], this is only the second study in which genetic influences on PTSD were examined in children and adolescents. Specifically, three SNPs 
Table 4

Latent growth curve analysis results

\begin{tabular}{lrrrr}
\hline & B & S.E.(B) & B/S.E.(B) & StdYX \\
\hline Intercept & & & & \\
African American & 0.26 & 2.59 & 0.10 & 0.02 \\
Other NonWhite & 1.59 & 3.75 & 0.42 & 0.07 \\
Age & -0.01 & 0.41 & -0.03 & -0.01 \\
Days in Hospital & -0.18 & 0.19 & -0.93 & -0.13 \\
Injury Severity Score & 0.26 & 0.24 & 1.10 & 0.22 \\
Violent Injury & -1.99 & 2.17 & -0.64 & -0.15 \\
Female & 6.83 & 2.81 & $2.43^{*}$ & 0.39 \\
rs12944712 & 5.77 & 1.72 & $3.35^{*}$ & 0.52 \\
Slope & & & & \\
African American & 0.22 & 0.17 & 1.3 & 0.23 \\
Other NonWhite & 0.18 & 0.20 & 0.90 & 0.13 \\
Age & 0.02 & 0.02 & 1.12 & 0.15 \\
Days in Hospital & 0.03 & 0.02 & 1.75 & 0.36 \\
Injury Severity Score & -0.03 & 0.02 & -1.40 & -0.31 \\
Violent Injury & 0.91 & 0.27 & $3.33^{*}$ & 0.86 \\
Female & 0.13 & 0.23 & 0.56 & 0.86 \\
rs12944712 & -0.42 & 0.11 & $-3.74^{*}$ & -0.58 \\
\hline
\end{tabular}

Note. $\mathrm{B}=$ Estimate; $\mathrm{SE}(\mathrm{B})=$ standard error of estimate; $\mathrm{B} / \mathrm{SE}(\mathrm{B})=\mathrm{z}$ statistic; ${ }^{*} p<0.01 ; \operatorname{StdXY}=$ standardized increase in $\mathrm{Y}$ given a standard deviation increase in $\mathrm{X}^{*} p<0.01$.

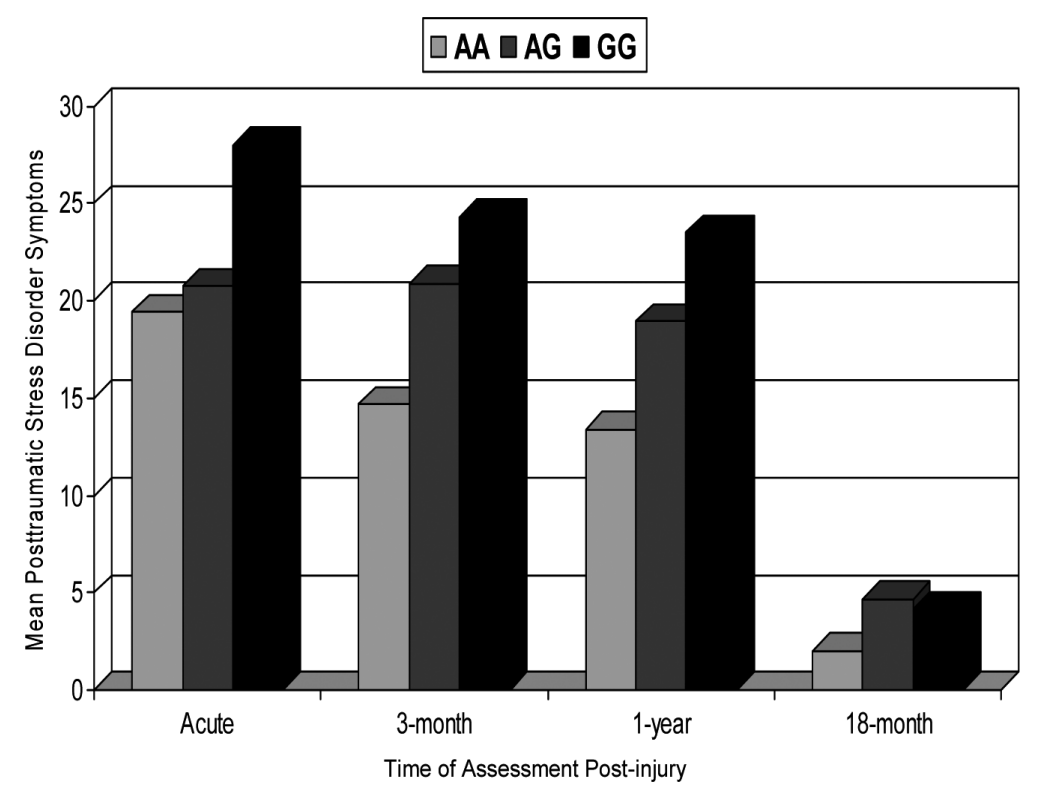

Fig. 2. Mean PTSD-RI symptoms by rs12944712 genotype.

(rs4074461, rs12944712, rs17763104) were related to PTSD symptoms on a univariate level, and after correction for multiple testing, rs 12944712 remained significant. For rs 1294412, the minor allele (A) was protective with regard to level of PTSD symptoms reported at the acute hospital visit, and given that self-reported race/ethnicity was not related to PTSD symptoms at any time point, it is unlikely that this was due to issues related to population stratification.
In contrast to the univariate analysis, the longitudinal analysis controlled for potential individual level influences (i.e., age, sex, self-reported race/ethnicity) and injury characteristics (i.e., injury severity, days in the hospital, whether the injury was violent in nature). With regard to covariates, elevations in initial PTSD symptom levels was predicted only by female gender, which was unrelated to subsequent course of symptoms. The only covariate found to significantly pre- 
dict subsequent symptom course was having sustained a violent injury; youth sustaining injuries secondary to violence evidenced significantly more symptoms over time than youth who sustained nonviolent injuries. Interestingly, growth analyses revealed significant effects of rs1294412 on both onset and course of PTSD symptoms. Consistent with univariate analyses, the minor allele of rs1294412 was associated with lower acute PTSD symptoms, again suggesting a protective effect. However, interestingly, the major allele $(G)$ was associated with a significantly greater decline of symptoms over time. It is possible that those with the major allele (G) had higher symptoms acutely and therefore had more opportunity for a sharper decline over the course of the study. Alternatively, it is possible that children exhibiting the most acute distress were also more likely to receive attention from family and medical personnel and that this support mitigated symptoms over time. This is a question we hope to explore in future studies.

Although this is the first examination of $C R H R 1$ genetic variation in relation to PTSD, our findings related to the degree of PTSD symptoms are generally consistent with previous studies of examining depression in which protective effects of certain CRHRI SNPs (e.g., rs110402, TAT haplotype) in individuals with a child abuse history have been reported $[42,43]$. Our rs12944712 SNP is in strong LD with three of the four SNPs found to be protective these in prior reports (rs7209436, rs110402, rs242924). Given that rs12944712 is an intronic SNP with no known function, future research is needed to determine if this SNP is causally related, or if it is just in high LD with a functional SNP. In other words, the specific biological mechanism via which the minor allele of rs 12944712 in CRHR l may be associated with decreased risk of PTSD symptoms acutely, but be associated with a slower decline in symptoms over time remains to be elucidated.

Although our sample size was too small to fully examine specific sex effects, previous reports of CRHRI variation suggest that the genes' protective effect of buffering against depression in adulthood in those with a history of child abuse may be specific to men [21], however this finding is not universally replicated [43]. The relatively consistent finding across these CRHRI investigations with our results is rather remarkable, given the major methodologic differences (e.g., different SNPs genotyped, all previous papers examined adults, the majority of traumatic events experienced in the previous studies were physical or sexual assault). Taken together, these findings point towards relevant polymorphisms for stress-related psychopathology in the CRHRl gene.
Although the specific functional variants in the CRHRI gene and their downstream effects are yet to be discovered, the biologic relevance of this gene as a whole to stress-related psychopathology such as PTSD is clear in light of the key role that CRH has on activating the HPA axis. Given evidence that PTSD patients hypersuppress cortisol in response to low-dose dexamethasone treatment [44], models of PTSD development have been expanded to incorporate altered posttrauma cortisol response. Yehuda and colleagues proposed that exaggerated catecholamine increases during traumatic stress without the regulatory influence of accompanying cortisol increases could lead to inappropriate memory formation (either over-salient or fragmented memories) and result in the intrusion symptoms that characterize PTSD [45]. It is possible that variation in CRHRl plays a role in this process, although that is an empirical question not yet answered. Nonetheless, the importance of $C R H R I$ in stress-related pathology is supported by preclinical and clinical studies $[4,5]$.

\subsection{Limitations and future directions}

The present data, although unique, are not without limitations. First, the study was limited by its small sample size and attrition over the course of follow-up assessments. Due to the small sample size we were under-powered to detect small effects, and therefore results should be considered preliminary. This also limited our ability to fully examine GxE interactions, possible developmental specific effects, and possible sex effects. Further, estimates derived from small samples may not be stable. Clearly, replication in a larger sample is warranted. Second, this investigation examined PTSD symptoms, not a clinical diagnosis of PTSD; therefore generalization to the PTSD diagnosis may be limited. Additionally, as noted above, HPA-axis dysregulation is not specific to PTSD, which was the only phenotype examined in the present paper. Future research should also examine CRHRl variation in relation to other mood and anxiety disorders. Third, the results may be confounded by population stratification. However, genotype distribution did not differ by racial group and self-reported racial/ethnic status was not related to PTSD symptoms, so this is unlikely. Fourth, although 8 SNPs were analyzed, only $58-62 \%$ of the variation in the CRHRl gene was accounted for. Future studies of this gene would be strengthened by fine mapping and sequencing, thereby allowing for identification of functional variants. Lastly, although a stressexposed cohort study has advantages, generalizabili- 
ty of the results may be limited as the only traumatic event category examined in the present study was injuries. Future studies should examine a range of traumatic event experiences to determine if the findings in the present study hold.

\subsection{Conclusions}

Our results suggest that $C R H R I$ variation is related to PTSD symptoms in pediatric injury patients, both acutely, and longitudinally. This is the first longitudinal genetic association study of PTSD symptoms in children that we are aware of that examines gene-symptom relations within a sample that was recently exposed to a significant stressor. Furthermore, this is the first examination of CRHRI SNPs in association with PTSD in a trauma-exposed sample, and it is also the second study to examine PTSD in children and adolescents. The prospective nature of our study makes our findings less vulnerable to ascertainment or recall biases than for studies that retrospectively assess adults regarding childhood experiences. Results from this study are not definitive and additional research is needed to replicate our methodology and findings, but our results suggest that studying an acutely-exposed hospitalized sample is both feasible and may improve power to find gene-disorder associations. Moreover, our findings in conjunction with data from preclinical models [4] and the literature on CRHR1 and stress-related pathology in humans $[42,43,46]$ suggest that this gene is indeed implicated in the post-trauma trajectory.

\section{Acknowledgements}

Dr. Amstadter is supported by US-NICHD HD0558 85. Dr. Nugent is supported by US-NIMH K01 MH 087240. Dr. Yang is supported by US-NIDA K01 DA 024758. Dr. Koenen is supported by US-NIMH K08 MH070627 and a Junior Faculty Sabbatical from the Harvard School of Public Health. This work was also supported by US-NIH grants MH078928, MH063247, MH086309 and the Robert Wood Johnson Foundation.

\section{References}

[1] S.J. Claes, Corticotropin-releasing hormone (CRH) in psychiatry: from stress to psychopathology, Ann Med 36 (2004), 50-61.

[2] R. Yehuda, Status of Glucocorticoid Alterations in Posttraumatic Stress Disorder, Annals of the New York Academy of Sciences 1179 (2009), 56-69.
[3] K.C. Koenen, Genetics of posttraumatic stress disorder: Review and recommendations for future studies, Journal of Traumatic Stress 20 (2007), 737-750.

[4] M.M. van Gaalen et al., Effects of transgenic overproduction of $\mathrm{CRH}$ on anxiety-like behaviour, The European journal of neuroscience 15 (2002), 2007-2015.

[5] D.G. Baker et al., Higher Levels of Basal Serial CSF Cortisol in Combat Veterans With Posttraumatic Stress Disorder, Am J Psychiatry 162 (2005), 992-994.

[6] J. Smoller et al., The corticotropin releasing hormone gene and behavioral inhibition in children at risk for panic disorder, Biological Psychiatry 57 (2005), 1485-1492.

[7] A.R. Tyrka et al., Interaction of Childhood Maltreatment with the Corticotropin-Releasing Hormone Receptor Gene: Effects on Hypothalamic-Pituitary-Adrenal Axis Reactivity, Biological Psychiatry 66 (2009), 681-685.

[8] M.C. Cornelis et al., Genetics of Posttraumatic Stress Disorder: Review and recommendations for genome-wide association studies, Current Psychiatry Reports, in press.

[9] A.W. Bachmann et al., Glucocorticoid receptor polymorphisms and post-traumatic stress disorder, Psychoneuroendocrinology 30 (2005), 297-306.

[10] E.B. Binder et al., Association of FKBP5 polymorphisms and childhood abuse with risk of posttraumatic stress disorder symptoms in adults, JAMA (Journal of the American Medical Association) 299 (2008), 1291-1305.

[11] S.S. Drury et al., The role of the dopamine transporter (DAT) in the development of PTSD in preschool children, $J$ Trauma Stress 22 (2009), 534-539.

[12] D.L. Delahanty ed., The Psychobiology of Trauma, 2008, Jason Aronson. 288.

[13] C.F. Gillespie et al., Risk and resilience: genetic and environmental influences on development of the stress response, Depress Anxiety 26 (2009), 984-992.

[14] P. Pervanidou, G.P. Chrousos and M. Luciano, Neuroendocrinology of Post-Traumatic Stress Disorder, in Progress in Brain Research, Elsevier. pp. 149-160.

[15] P. Pervanidou, Biology of post-traumatic stress disorder in childhood and adolescence, Journal of Neuroendocrinology 20 (2008), 632-638.

[16] D.L. Delahanty, A.J. Raimonde and E. Spoonster, Initial posttraumatic urinary cortisol levels predict subsequent PTSD symptoms in motor vehicle accidents, Biological Psychiatry 48 (2000), 940-947.

[17] S.A. Ostrowski et al., Acute child and mother psychophysiological responses and subsequent PTSD symptoms following a child's traumatic event, Journal of Traumatic Stress 20 (2007), 677-687.

[18] D.L. Delahanty et al., Initial urinary epinephrine and cortisol levels predict acute PTSD symptoms in child trauma victims, Journal of Psychoneuroendocrinology 30 (2005), 121-128.

[19] P. Pervanidou et al., The Natural History of Neuroendocrine Changes in Pediatric Posttraumatic Stress Disorder (PTSD) After Motor Vehicle Accidents: Progressive Divergence of Noradrenaline and Cortisol Concentrations Over Time, Biological Psychiatry 62 (2007), 1095-1102.

[20] C.F. Weems and V.G. Carrion, The association between PTSD symptoms and salivary cortisol in youth: The role of time since the trauma, Journal of Traumatic Stress 20 (2007), 903-907.

[21] C. Heim et al., Effect of childhood trauma on adult depression and neuroendocrine function: sex-specific moderation by CRH receptor 1 gene, Frontiers in Behavioral Neuroscience 3 (2009). 
[22] R.G. Bradley et al., Influence of Child Abuse on Adult Depression: Moderation by the Corticotropin-Releasing Hormone Receptor Gene, Arch Gen Psychiatry 65 (2008), 190-200.

[23] G. Polanczyk et al., Protective Effect of CRHR1 Gene Variants on the Development of Adult Depression Following Childhood Maltreatment: Replication and Extension, Arch Gen Psychiatry 66 (2009), 978-985.

[24] K. Handwerger, Differential patterns of HPA activity and reactivity in adult posttraumatic stress disorder and major depressive disorder, Harvard Reviews Psychiatry 17 (2009), 184 205.

[25] N.R. Nugent, A.B. Amstadter and K.C. Koenen, Genetics of PTSD: Informing Clinical Conceptualizations and Promoting Future Research, American Journal of Medical Genetics C seminars in Medical Genetics 148 (2008), 127-132.

[26] G.N. Saxe et al., Incidence of and risk factors for acute stress disorder in children with injuries, J Trauma 59 (2005), 946953.

[27] G. Saxe et al., Separation anxiety as a mediator between acute morphine administration and PTSD symptoms in injured children, Annals of the New York Academy of Sciences 1071 (2006), 41-45.

[28] K.C. Koenen et al., Polymorphisms in FKBP5 are associated with peritraumatic dissociation in medically injured children, Mol Psychiatry 10 (2005), 1058-1059.

[29] K.A. Frazer et al., A second generation human haplotype map of over 3.1 million SNPs, Nature 449 (2007), 851-861.

[30] J.C. Barrett et al., Haploview: analysis and visualization of LD and haplotype maps, Bioinformatics 21 (2005), 263-265.

[31] P.I. de Bakker et al., Efficiency and power in genetic association studies, Nat Genet 37 (2005), 1217-1223.

[32] R.S. Pynoos et al., UCLA PTSD index for DSM-IV (child, adolescent, and parent version) UCLA Trauma Psychiatry Service, (1998).

[33] N. Rodriguez, A.M. Steinberg and R.S. Pynoos, UCLA PTSD Index for DSM-IV instrument information: Child, Adolescent, and Parent Version, Los Angelos: UCLA Trauma Psychiatry Service, (1998).

[34] A.M. Steinberg et al., The University of California at Los Angeles post-traumatic stress disorder reaction index, Current Psychiatry Reports 6 (2004), 96-100.
[35] A. Roussos et al., Posttraumatic stress and depressive reactions among children and adolescents after the 1999 earthquake in Ano Liosia, Greece, American Journal of Psychiatry 162 (2005), 530-537.

[36] S.P. Baker et al., The injury severity score: A method for describing patients with multiple injuries and evaluating emergency care, Journal of Trauma 14 (1974), 187-196.

[37] S. Purcell et al., PLINK: a tool set for whole-genome association and population-based linkage analyses, Am J Hum Genet 81 (2007), 559-575.

[38] M.P. Epstein and G.A. Satten, Inference on haplotype effects in case-control studies using unphased genotype data, American Journal of Human Genetics 73 (2003), 1316-1329.

[39] M.D. Li, J.Z. Ma and J. Beuten, Progress in searching for susceptibility loci and genes for smoking-related behaviour, Clin Genet 66 (2004), 382-392.

[40] T.E. Duncan and S.C. Duncan, An introduction to latent growth curve modeling, Behavior Therapy 35 (2004), 333363.

[41] L.K. Muthen and B.O. Muthen, Mplus Version 3.11. 2004, Muthen and Muthen: Los Angeles, CA, (2004).

[42] R.G. Bradley et al., Influence of child abuse on adult depression moderation by the corticotropin-releasing hormone receptor gene Archives of General Psychiatry 65 (2008), 190200.

[43] G. Polanczyk et al., Protective effect of CRHR1 gene variants on the development of adult depression following childhood maltreatment. Replication and extension, Archives of General Psychiatry 66 (2009), 978-985.

[44] R. Yehuda et al., Dose response changes in plasma cortisol and lymphocyte glucocorticoid receptors following dexamethasone administration in combat veterans with and without PTSD, Archives of General Psychiatry 52 (1995), 583-593.

[45] R. Yehuda, A.C. McFarlane and A.Y. Shalev, Predicting the development of posttraumatic stress disorder from the acute response to a traumatic event, Biol Psychiatry 44 (1998), 13051313.

[46] C. Heim et al., Effect of childhood trauma on adult depression and neuroendocrine function: sex-specific moderation by $\mathrm{CRH}$ receptor 1 gene, Frontiers in Behavioral Neuroscience 41 (2009), 1-10. 


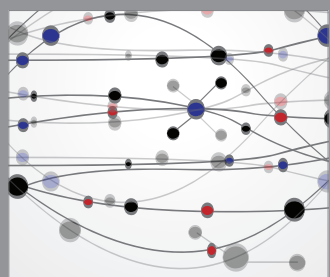

The Scientific World Journal
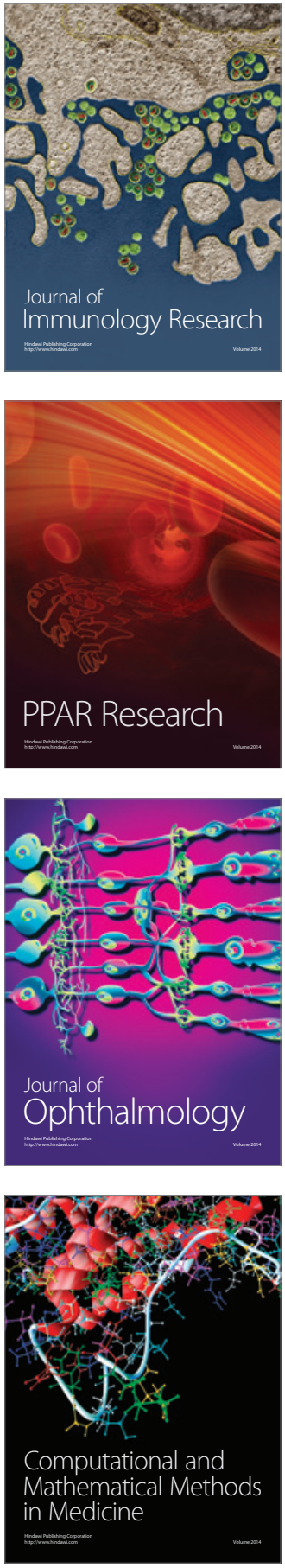

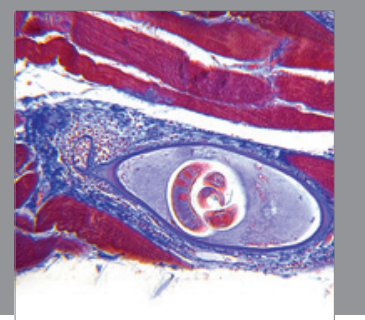

Gastroenterology

Research and Practice
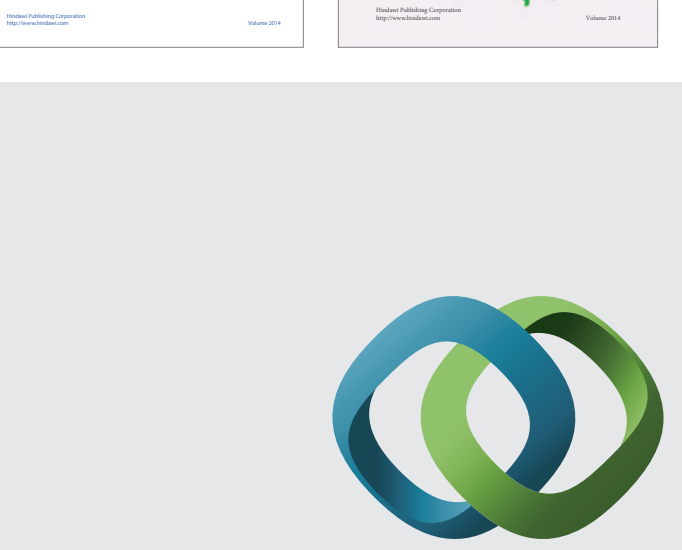

\section{Hindawi}

Submit your manuscripts at

http://www.hindawi.com
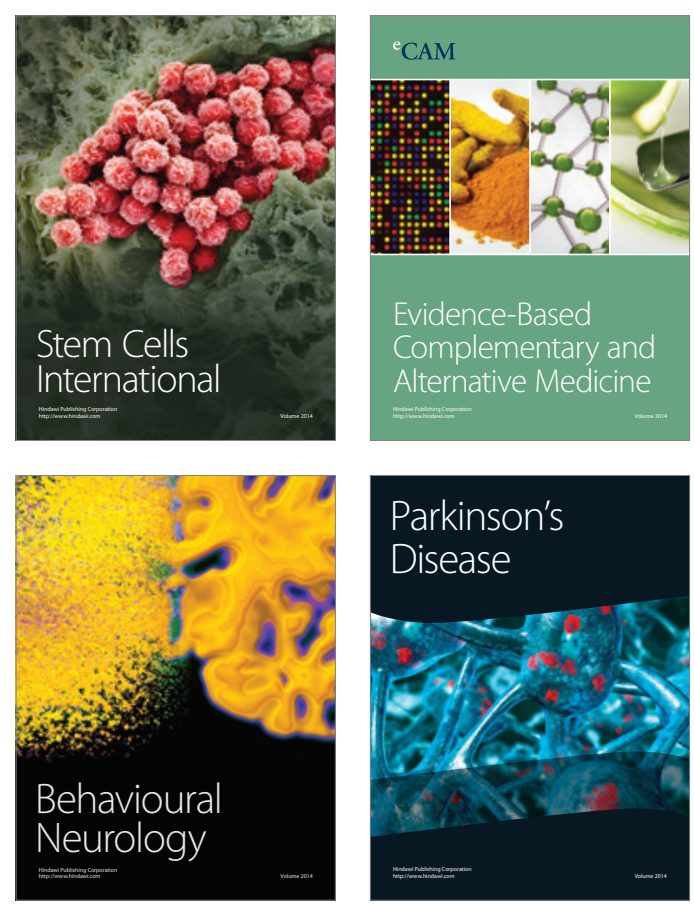

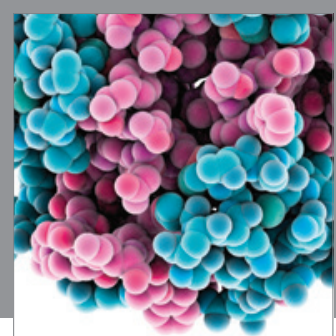

Journal of
Diabetes Research

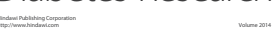

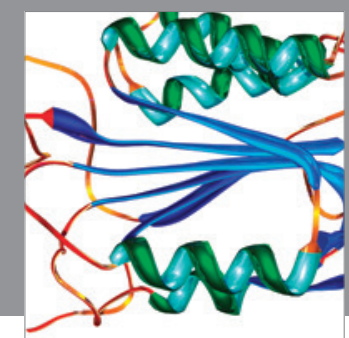

Disease Markers
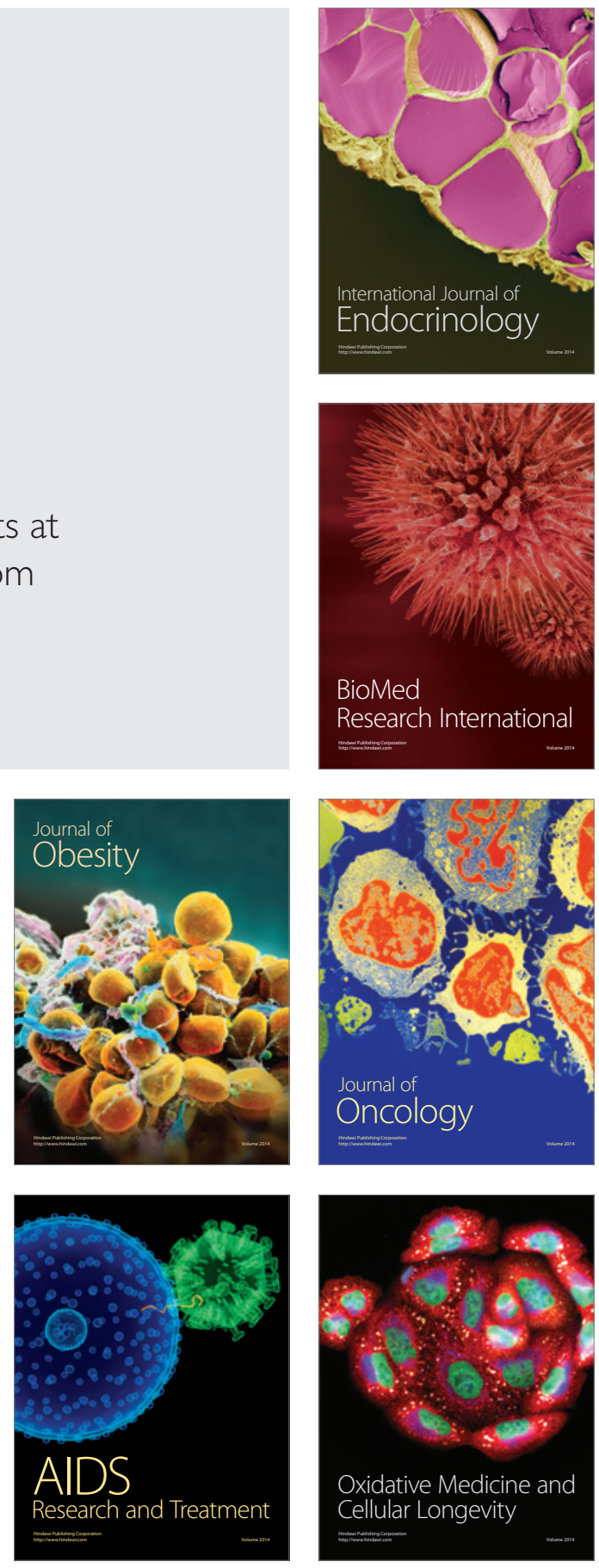